\title{
Influência da suplementação nutricional sobre 0 metabolismo energético e enzimas musculares em cavalos de Team Penning
}

\author{
Influence of nutritional supplementation on energetic \\ metabolism and muscle enzymes in Team Penning horses
}

Eriky Akio de Oliveira Tongu, Raissa Oliveira Leite, Amanda Bizare, Isadora Araújo Naves, Kamila Pinheiro Paim, Larissa Freitas Campos, Antonio Vicente Mundim, João Paulo Elsen Saut, Diego José Zanzarini Delfiol

Faculdade de Medicina Veterinária, Universidade Federal de Uberlândia (UFU), Uberlândia, MG, Brasil

\section{Resumo}

0 Team Penning (TP) é uma modalidade equestre que exige velocidade máxima. Nesse tipo de exercício ocorre o rápido consumo de energia pela via anaeróbica. Diversos suplementos têm sido desenvolvidos para melhorar o desempenho e diminuir lesões musculares em equinos atletas. Objetivou-se neste estudo avaliar a influência da suplementação com Tonnus JCR ${ }^{\circledR}$ nos parâmetros fisiológicos, metabolismo energético e muscular no treinamento de TP. Foram utilizados 12 equinos da raça Quarto de Milha, atletas de TP, separados em dois grupos, suplementado (GS) e não suplementado (GNS), avaliados em dois treinos. Foi realizado o exame físico, avaliação das concentrações plasmáticas de glicose e lactato, e concentrações séricas de creatinoquinase (CK), aspartato aminotransferase (AST) e lactato desidrogenase (LDH). As amostras de sangue foram colhidas durante e após os treinos em 12 diferentes momentos (M0 - M11). A suplementação foi realizada no intervalo entre os treinos. No segundo treino, verificou-se diminuição nas concentrações de glicose no GS quando comparado ao treino $1(p=0,01)$; no GNS não houve diferença entre os treinos $(p=0,13)$. Não houve diferença nas concentrações de lactato entre grupos e treinos. Os valores da concentração de CK no treino 1 não apresentaram diferença entre os grupos $(\mathrm{p}=0,83)$ e momentos $(\mathrm{p}=$ $0,16)$. Quando comparadas as concentrações de CK entre os grupos no treino 2, observaram-se concentrações menores no GS $(p=0,02)$. Ao se comparar o GS antes e após a suplementação, as concentrações de CK foram menores no treino 2 ( $p=0,01)$. No GNS não houve diferença entre os treinos $(p=0,51)$. As enzimas AST e LDH não diferiram entre os grupos nos treinos 1 e 2 . Concluiu-se que a suplementação influenciou de forma positiva, diminuindo as concentrações de glicose e os níveis de $\mathrm{CK}$, o que indica menor injúria muscular pós-treino.

Palavras-chave: Lesão muscular. Lactato. Energia anaeróbica. Cavalo atleta. 


\section{Abstract}

Team Penning (TP) is an equestrian sport that requires maximum speed, in which there is rapid energy consumption via the anaerobic pathway. Several supplements were developed to improve performance and decrease muscle injuries in athletic horses. The objective of this study was to evaluate the influence of Tonnus JCR ${ }^{\circledR}$ supplementation on physiological parameters, energy metabolism and muscle in horses undergoing TP training. Twelve Quarter breed horses were used; TP animals were separated into two groups, supplemented (GS) and non-supplemented (GNS), and were evaluated in two training sessions. Physical examination was performed to evaluate the concentration of glucose and plasma lactate, and the serum levels of creatine kinase (CK), aspartate aminotransferase (AST), and lactate dehydrogenase (LDH). Blood samples were collected during and after training at 12 different moments (MO - M11). Supplementation to the GS horses was done during training intervals with Tonnus JCR ${ }^{\circledR}$. At the second training session, there was a decrease in glucose values in GS animals when compared to the first training moment ( $p=0.01$ ); whereas in GNS horses there was no difference ( $p=0.13$ ). There were no differences in lactate levels between groups and training sessions. $C K$ values in training 1 presented no difference between the groups ( $p=0.83)$ and moments ( $p=0.16)$. Lower levels in G1 ( $p=0.02)$ were observed when the CK levels were compared between the groups at training 1. When GS animals were compared before and after supplementation, CK levels were lower in the second training session $(p=0.01)$. No difference between training sessions ( $p=0.51$ ) was observed in GNS horses. Differences in the enzymatic activity of AST and LDH were not observed between the groups of horses in both training sessions. It was concluded that supplementation had a positive effect, resulting in decreased concentrations of glucose and the activity of $\mathrm{CK}$, suggesting less muscular damage.

Keywords: Muscle injury. Lactate. Anaerobic energy. Athletic horses.

\section{Introdução}

Dentre os esportes equestres, o Team Penning é uma modalidade que exige muita técnica, velocidade e agilidade dos cavalos. Neste tipo de exercício, que envolve velocidade máxima em curta duração, os depósitos de energia nas fibras musculares são consumidos rapidamente pela via anaeróbica, favorecendo o acumulo de ácido lático e a presença de amoníaco, que contribuem para a lesão muscular (Richardson e Murray, 2016).

A avaliação da composição bioquímica sanguínea reflete de forma precisa a situação metabólica dos diversos tecidos, permitindo avaliar lesões teciduais e transtornos no funcionamento de órgãos, adaptação do animal diante de desafios nutricionais e fisiológicos, e desequilíbrios metabólicos. Essas alterações metabólicas, mesmo que pequenas, podem refletir processos capazes de reduzir substancialmente o potencial atlético do animal (Franklin e Allen, 2014).

A glicose é o principal metabolito utilizado para obtenção de energia pela musculatura esquelética, sendo regulada pela atividade hormonal do cortisol, adrenalina e glucagon que, ao serem liberados, provocam glicogenólise e gliconeogênese hepática, aumentando, assim, a biodisponibilidade da glicose durante o exercício (Ferraz et al., 2010; Santiago et al., 2013).

A concentração plasmática de lactato tem sido estudada em testes controlados na medicina equina na tentativa de quantificar a intensidade do exercício, sendo utilizada para estabelecer os níveis de condicionamento físico, podendo ser um indicador da capacidade cardiovascular e metabólica dos cavalos atletas (Malblanc e van Erck-Westergren, 2014). Durante o exercício, o aumento da concentração de lactato na musculatura e no sangue se deve à interação de uma série de processos fisiológicos e bioquímicos, dentre eles a baixa disponibilidade de oxigênio em situações de exercício submáximo (Gladden, 2004; Gillespie et al., 2017).

As alterações musculares possuem sinais clínicos semelhantes e inespecíficos e, por isso, somente a observação destes não é suficiente para o diagnóstico, sendo necessária a utilização de exames complementares como a dosagem da atividade enzimática da creatina quinase (CK), aspartato aminotransferase (AST) e lactato desidrogenase (LDH) (Trigo et al., 2010; Franklin e Allen, 2014).

0 comércio e a utilização de suplementos nutricionais em cavalos de esporte com o intuito de melhorar o desempenho atlético desses animais 
tem crescido nos últimos anos, entretanto seu uso ainda é controverso e apresenta limitada evidência científica (Harris e Harris, 2005; Swirsley et al., 2017).

Alguns ingredientes presentes nos suplementos comerciais, como a L-carnitina (Sato et al., 2015), aminoácidos de cadeia ramificada (BCAAs) (Harris e Harris, 2005), gama-orizanol (Ostaszewski et al., 2012), cromo quelato (Jordão et al., 2010), ômega 3, pré e próbiótico (Urschel e Lawrence, 2013) e creatina (Teixeira et al., 2016), podem auxiliar na diminuição de lesões musculares induzidas pelo exercício, no aumento de energia disponível e na redução da fadiga. Entre os suplementos comerciais, destaca-se o Tonnus JCR ${ }^{\circledR}$ por possuir esses componentes na sua formulação, sendo desenvolvido para equinos com o intuito de melhorar o desempenho atlético, acelerar o processo de recuperação entre um exercício e outro, além de diminuir os riscos de lesão muscular (Vetnil, 2012).

O objetivo desse trabalho foi avaliar a influência da suplementação com o Tonnus JCR ${ }^{\circledR}$ nos parâmetros físicos e nas concentrações de glicose, lactato, CK, AST e LDH em cavalos atletas de Team Penning.

\section{Material e métodos}

\section{Animais, local e manejo}

Esse experimento foi aprovado pela Comissão de Ética na Utilização de Animais da Universidade Federal de Uberlândia, sob o número de protocolo 070/16, e realizado em um centro de treinamento localizado na cidade de Uberlândia, MG.

Foram selecionados 12 equinos da raça Quarto de Milha, sendo seis fêmeas e quatro machos, vermifugados e vacinados para encefalomielite equina leste e oeste, influenza equina, rinopneumonite, herpes vírus equino tipo 1 e 4 e tétano. Os animais foram previamente divididos de forma aleatória em dois grupos: suplementado (GS) e não suplementado (GNS).

Durante todo o período experimental, cada equino foi mantido em baias de alvenaria medindo $16 \mathrm{~m}^{2}$, com ração fornecida em duas porções de $2 \mathrm{~kg}$ (Supra ${ }^{\circledR}$ ProCavalo melaçada) em cada período do dia (manhã e tarde), feno de coast-cross (Cynodon dactylon) e água ad libitum.

\section{Critérios de inclusão e exclusão inicial e durante o experimento}

Foram utilizados como critério de inclusão para seleção dos equinos animais hígidos, com hemograma dentro da normalidade e já adaptados a treinamentos e competições de Team Penning há pelo menos um ano, e que não estivessem recebendo qualquer tipo de suplementação além de feno e ração.

Foram adotados como critério de exclusão animais que apresentaram miopatias, claudicação e/ ou doenças sistêmicas que pudessem comprometer os resultados.

\section{Delineamento experimental}

Inicialmente, os animais que atenderam aos critérios de inclusão foram divididos aleatoriamente em dois grupos: GS $(n=6)$ e GNS $(n=6)$. 0 experimento contou com o acompanhamento de dois treinos consecutivos, denominados de treinos 1 e 2 , com intervalo de 80 dias.

Em cada treino, os cavalos realizavam um aquecimento prévio de 30 minutos, dividido em: $10 \mathrm{~min}$ a passo, $10 \mathrm{~min}$ a trote, $5 \mathrm{~min}$ de galope e $5 \mathrm{~min}$ a passo. Após o aquecimento, cada animal realizava 10 participações em pista, denominadas "passadas". Cada passada possuía um tempo médio de 18 segundos. Entre cada passada os animais ficavam em repouso por um tempo de $5 \mathrm{~min}$. Após o término do treino, os animais foram desencilhados e colocados em baia. Durante o intervalo de 80 dias entre os treinos avaliados, os animais continuaram executando estas atividades duas vezes por semana, às terças e quintas-feiras.

Em cada um dos dois treinos acompanhados foi realizado o exame físico e coleta de sangue. Após o término do treino 1, os cavalos do GS foram suplementados com 50 gramas de Tonnus JCR ${ }^{\circledR}$ pó adicionado à ração duas vezes ao dia, totalizando 100 gramas por animal/dia, durante 80 dias (intervalo entre os treinos 1 e 2). Cinco dias antes da realização do treino 2 , os cavalos do GS passaram a receber, além do Tonnus $\mathrm{JCR}^{\circledR}$ pó, 20 gramas de Tonnus JCR $^{\circledR}$ pasta, conforme recomendação do fabricante. Os cavalos do GNS não receberam nenhuma destas duas suplementações. 


\section{Exame físico e coleta das amostras}

Foi realizado exame físico dos animais, avaliação da atividade sérica das enzimas CK, AST e LDH, e da concentração plasmática de lactato e glicose. No exame físico foram avaliadas a frequência respiratória e cardíaca, tempo de preenchimento capilar (TPC), coloração de mucosa e temperatura retal de acordo com Feitosa (2014). Quando necessário, exame físico específico do sistema locomotor (Stashak, 2006). As amostras de sangue foram coletadas por venopunção da jugular externa em sistema a vácuo, com agulha $21 \mathrm{G}$, utilizando tubos sem anticoagulante para obtenção de soro para as análises de CK, AST e LDH, e tubos contendo fluoreto de sódio para obtenção de plasma para a dosagem de lactato e glicose.

As amostras foram coletadas nos treinos 1 e 2 em 12 diferentes momentos (M): em repouso (M0); imediatamente após o animal realizar uma passada (M1); após duas passadas (M2); após três passadas (M3); após seis passadas (M4); após oito passadas (M5); após dez passadas (M6); 30 minutos após o termino da décima passada (M7); após 6 horas (M8); após 12 horas (M9); após 24 horas (M10); após 48 horas (M11) do término do treino.

Os parâmetros fisiológicos da frequência cardíaca, frequência respiratória, TPC e coloração de mucosa foram avaliados do M0 ao M7; já a temperatura retal foi avaliada no M0 e no M7.

\section{Processamento e análise laboratorial}

Imediatamente após a coleta de sangue, as amostras foram centrifugadas a 720 g por cinco minutos para obtenção de soro para a dosagem de CK, AST e LDH e de plasma para determinação de glicose e lactato. As amostras de soro e plasma foram armazenas em tubos plástico WWs de 1,5 mL, refrigeradas e encaminhadas para o laboratório, onde as concentrações de CK, AST, LDH e glicose foram realizadas em até 12 horas após a coleta, e uma alíquota de plasma de cada cavalo do M0 ao M7 foi congelada a $-20{ }^{\circ} \mathrm{C}$ para posterior dosagem do lactato.

As concentrações de CK, AST, LDH, glicose e lactato foram realizadas utilizando kits comerciais Labtest ${ }^{\circledR}$, seguindo as recomendações do fabricante, com leitura no analisador automático de bioquímica ChemWell ${ }^{\circledR}$, periodicamente calibrado com Calibra $\mathrm{H}^{\circledR}$ e aferido com soro controle de valor conhecido Qualitrol ${ }^{\circledR}$.

\section{Análise Estatística}

A análise estatística foi realizada com o auxílio do programa Graphpad Prism Software v 6.03 (GraphPad Software Inc., La Jolla, CA) e a análise descritiva foi apresentada em média aritmética e desvio padrão. A frequência cardíaca (FC), frequência respiratória (FR) e as variáveis bioquímicas nos diferentes momentos foram avaliadas por análise de variância com dois fatores para medidas repetidas (Two-way ANOVA). Nas situações onde houve interação significativa, o pósteste de Bonferroni foi aplicado. Quando se realizou a comparação entre o mesmo grupo em treinos diferentes foi utilizado teste pareado, e quando se realizou a comparação entre os grupos foi realizado teste não pareado. 0 nível de significância para todos os testes foi de $95 \%$ ( $\mathrm{p} \leq 0,05)$.

\section{Resultados}

Os 12 equinos que atenderam aos critérios de inclusão apresentaram média de 7,4 $\pm 3,4$ anos e peso vivo de $369,5 \pm 70,5 \mathrm{Kg}$. Durante o experimento, dois equinos foram excluídos, sendo um do GS, diagnosticado com infecção ascendente da linha branca, e o outro do GNS, a pedido do proprietário. Não foram observadas alterações comportamentais ou efeito adverso da suplementação comercial fornecida aos animais.

Os parâmetros fisiológicos não apresentaram alterações nas avaliações nos momentos entre treinos e grupos. Em ambos os grupos os animais apresentavam as mucosas róseas e TPC de 2" em todas as avaliações. A temperatura retal média no treino 1 foi de $37,0 \pm 0,1^{\circ} \mathrm{C}$ no $\mathrm{M} 0$ e de $38,4 \pm 0,1^{\circ} \mathrm{C}$ no $\mathrm{M} 7$; já no treino $2,37,4 \pm 0,1^{\circ} \mathrm{C}$ no $\mathrm{M} 0$ e $38,5 \pm$ $0,1{ }^{\circ} \mathrm{C}$ no M7.

Em relação aos parâmetros FC e FR (Figura 1), não houve interação significativa entre os treinos 1 e 2 do GS e GNS, nem entre os grupos dentro dos treinos 1 e 2 . Além disso, não foram observadas 
diferenças significativas durante todo o período avaliado em relação às médias da $\mathrm{FC}$ entre os grupos, tanto no treino $1(\mathrm{p}=0,439)$ quanto no treino 2 ( $p=0$,132). Não se observou diferença, também, entre os treinos no GS ( $p=0,902)$ e no GNS ( $p=0,465)$. Em todas as situações (Figura 1
E-H) houve variação significativa em relação aos momentos. Em relação à $\mathrm{FR}$, não houve diferença no período avaliado entre os grupos no treino 1 ( $p=0,761)$ e no treino $2(p=0,227)$. Também não foram observadas diferenças entre os treinos no GS $(p=0,321)$ e no GNS $(p=0,481)$.

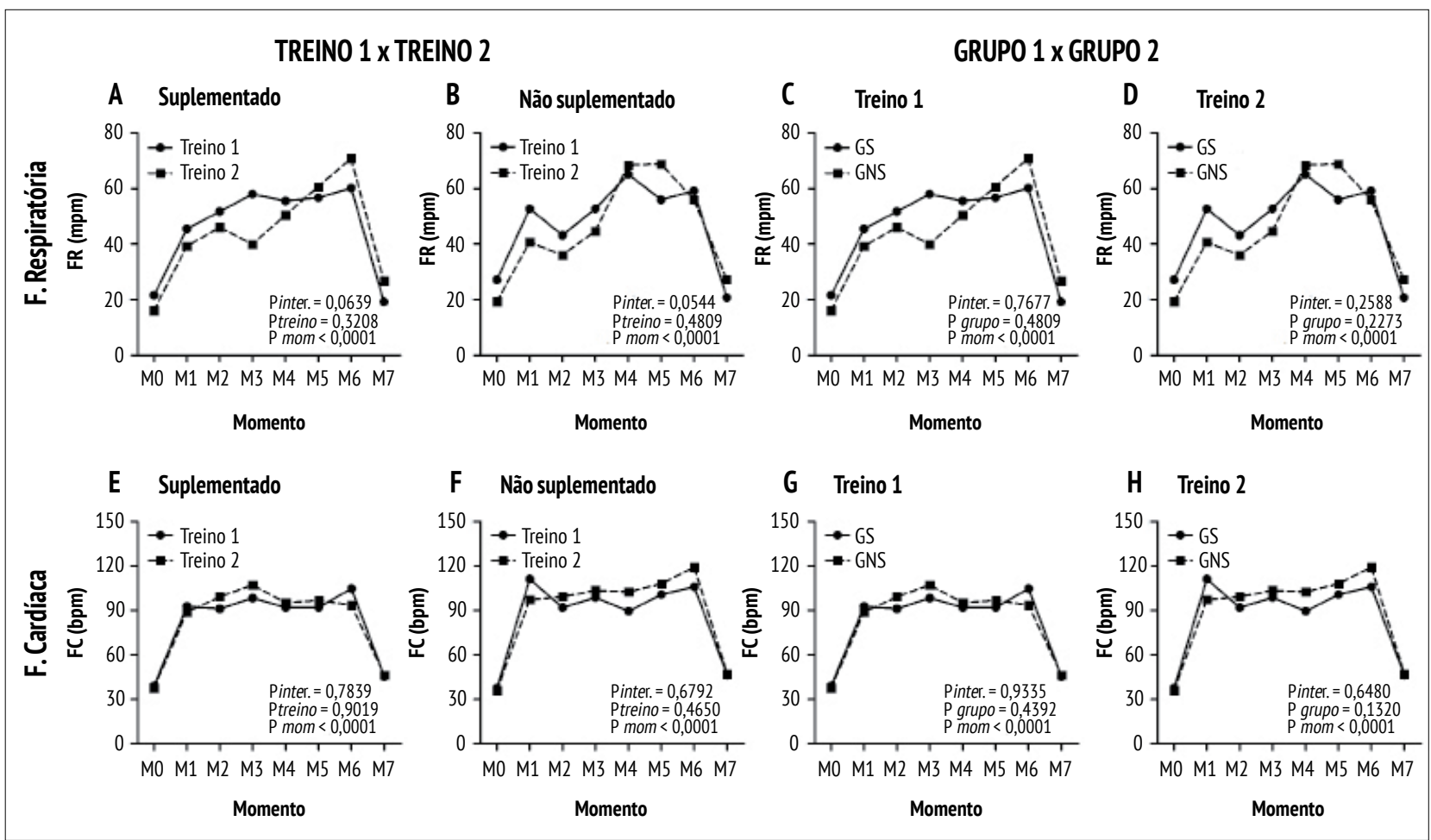

Figura 1 - Valores das mensurações de frequência cardíaca e respiratória nos diferentes momentos, treinos e grupos.

As concentrações plasmáticas de glicose e lactato estão apresentadas na Figura 2. Ao se considerar a média de todos os momentos, a concentração de glicose foi menor no GS durante o treino $1(\mathrm{p}=0,004)$ e não houve diferença significativa em relação ao lactato $(\mathrm{p}=0,170)$. No entanto, ao se comparar os treinos, houve diferença na concentração de glicose apenas no GS $(\mathrm{p}=0,016)$, ocorrendo uma redução na glicemia no segundo treino. Não foi observada diferença nas concentrações de lactato entre os grupos nos treinos, e não houve diferença entre os treinos no GS ( $p=0,582)$ e no GNS ( $p=0,698)$.

Em relação à concentração sérica da enzima AST (Figura 2), não houve diferença entre os grupos nos treinos $1(p=0,731)$ e $2(p=0,608)$. Quando se comparou os grupos antes e depois do período de suplementação, houve diferença entre os treinos ao se considerar todos os momentos, com valores menores no segundo treino no GNS. Observou-se interação dos momentos de coleta com os treinos no GS ( $\mathrm{p}<0,0001)$, com valores maiores de AST no M8 e no M11.

Os valores enzimáticos da CK (Figura 2) não apresentaram diferença entre os grupos $(\mathrm{p}=0,826)$ e momentos $(\mathrm{p}=0,16)$. Quando comparadas as concentrações de CK entre os grupos no treino 2 , observaram-se concentrações menores no GS ( $\mathrm{p}=0,02)$. Quando comparado o GS antes e após a suplementação, as concentrações de CK foram significativamente menores no treino $2(p=0,01)$. Já no GNS, não houve diferença estatística entre os treinos 1 e $2(p=0,511)$. 


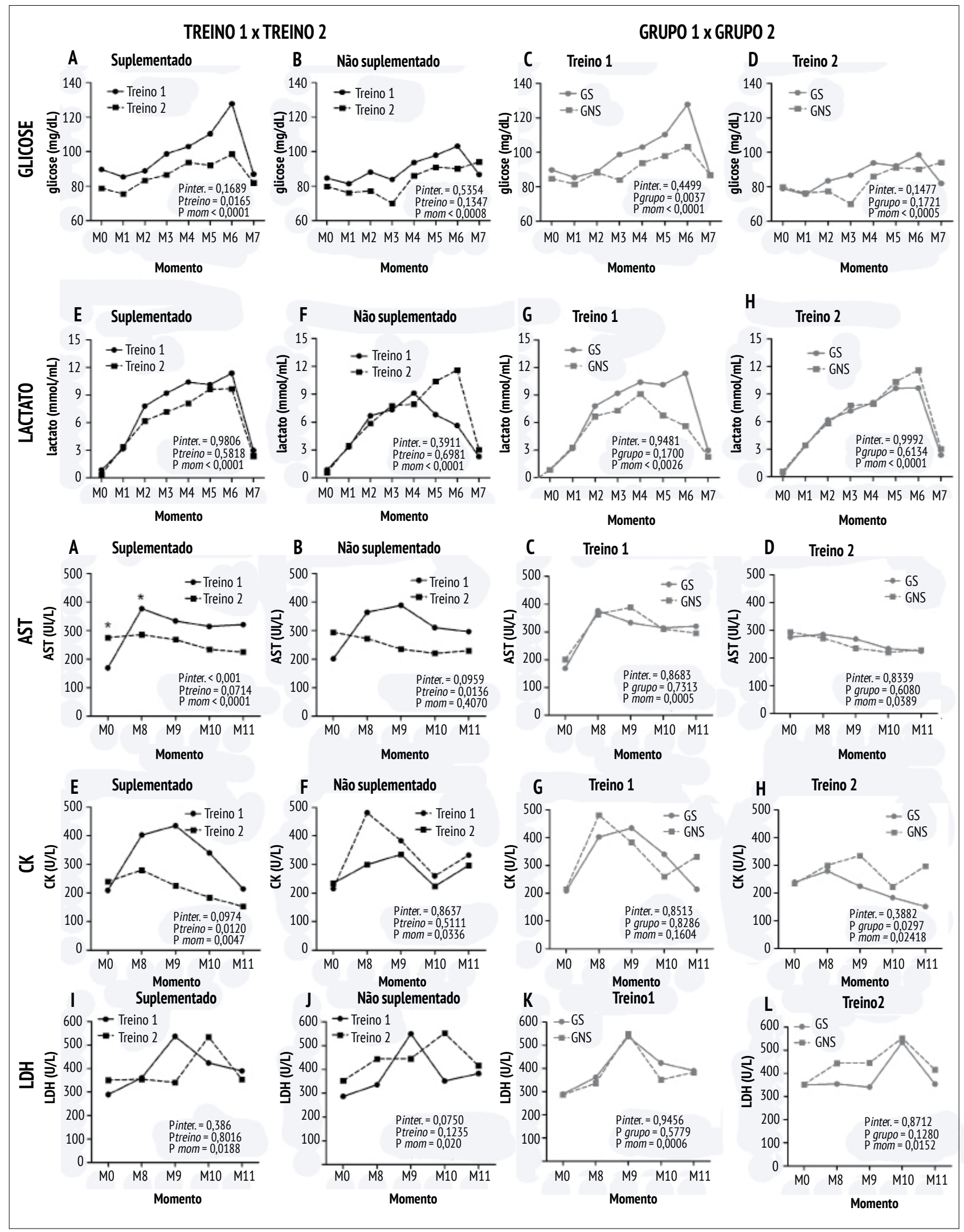

Figura 2 - Valores das concentrações plasmáticas de glicose e lactato e das concentrações séricas da enzimas creatina quinase (CK), aspartatoamino-transferase (AST) e lactato desidrogenase (LDH) nos diferentes momentos, treinos e grupos. 
As concentrações séricas de LDH (Figura 2) não demonstraram diferença entre os grupos nos treinos $1(p=0,578)$ e $2(p=0,128)$. Também não foram observadas diferenças nos grupos quando comparadas antes e depois da suplementação, tanto no GS ( $p=0,8016)$ quanto no GNS ( $p=0,123)$.

\section{Discussão}

FC e FR estavam elevadas logo após o M1; essas alterações são decorrentes do fator estresse e das compensações exigidas pelo organismo do animal para obtenção de energia durante o exercício (Becker-Birck et al., 2013). Outra explicação seria a estimulação simpática antes do início da competição, incluindo o processo de aquecimento, o que ocasiona um aumento na liberação de catecolaminas, principalmente adrenalina, elevando tanto a FR quanto a FC. Esses achados também estão correlacionados com o aumento da temperatura corporal durante o exercício, aumentando os mecanismos compensatórios de termorregulação (Hassan et al., 2015).

As concentrações de glicose diminuíram no grupo suplementado. Uma possível explicação seria que o cromo, juntamente com omega 3 , funcione como um fator de tolerância à glicose para auxiliar as funções de insulina, especificamente em relação ao metabolismo da glicose, anabolismo e ganho muscular (Vincent, 2007; Hess et al., 2013; Hodgson et al., 2014). O triptofano também se mostra importante na redução dos valores plasmáticos de glicose, reduzindo o estresse e, consequentemente, as concentrações de cortisol, como já demonstrado por Davis et al. (2017).

Os BCAAs possuem um papel na fadiga central, regulando o metabolismo do triptofano. Eles diminuem a absorção deste aminoácido, reduzindo as concentrações de serotonina no cérebro e, consequentemente, a fadiga (Harris e Harris, 2005; Votion et al., 2007). Embora tenha sido relatada uma leve diminuição nas contrações de lactato e glicose em cavalos suplementados com BCAAs (Stefanon et al., 2000), resultado semelhante ao encontrado no presente estudo, a admistração destes parece não afetar o desempenho no exercício.

Em exercícios de baixa intensidade, a suplemen- tação com BCAAs resultou em diminuição nos valores plasmáticos de lactato, entretanto, em exercícios de alta intensidade e com suplementação prolongada (4 a 5 semanas), não foram observadas reduções nas concentrações de lactato ou melhora no desempenho atlético (Urschel e Lawrence, 2013). Oliveira et al. (2014) demonstraram que diferentes quantidades de proteína na dieta não auxiliaram na diminuição da concentração de lactato plasmático. No presente estudo, apesar de não ocorrer diferença estatística, observou-se redução na concentração basal do lactato após a suplementação e metabolização mais rápida após o exercício.

O Tonnus JCR possui diversos componentes que atuam como antioxidantes, diminuindo, assim, espécies reativas a oxigênio (ROS) que são acumuladas durante o exercício intenso, resultando em danos musculares e fadiga (Hodgson et al., 2014). A diminuição significativa de CK no GS pode ter ocorrido devido ao menor acúmulo de ROS e, consequentemente, menor injúria muscular e melhor recuperação nos cavalos suplementados.

No treino 1 houve alterações musculares, demonstradas pelo rápido aumento de $\mathrm{CK}$ seguido por diminuição progressiva até o M11. Isso se confirma ao analisar as concentrações de AST e LDH - enzimas de meia vida mais longa, que auxiliam no diagnóstico de lesões musculares que se perpetuam, causando lesão de forma crônica (Ebge-Nwiyi et al., 2012) -, que apesar de terem sofrido um pequeno aumento no M8 para AST e no M9 para LDH, mantiveram-se dentro dos valores considerados normais para cavalos pós-exercício (Oliveira et al., 2014).

No treino 2, os animais do GS tiveram menores concentrações de CK, sem aumento de AST, apresentando, em ambas as enzimas, médias menores quando comparados com os animais do GNS e com os resultados antes da suplementação, o que indica menor dano muscular pós-exercício. Resultados semelhantes foram encontrados por Ostaszewski et al. (2012), que suplementaram cavalos de corrida com HMB (beta-hidroxi-beta-metilbutirato) e gamaorizanol e obtiveram diminuição nos níveis de CK e AST no grupo suplementado, concluindo uma diminuição nas lesões musculares pós-treino e provável aumento de desempenho. 
Um importante componente do suplemento fornecido é a creatina, um fosfato de alta energia utilizado pelos músculos como um doador para regenerar o ATP (trifosfato de adenosina) do ADP (difosfato de adenosina) nos estágios iniciais do exercício, mantendo o status energético, bem como a capacidade para tamponar os íons de hidrogénio produzidos durante exercício de alta intensidade, o que preveniria lesões musculares (Hodgson et al., 2014). Entretanto, a suplementação oral somente com a creatina em equinos não tem demostrado bons resultados (Schuback et al., 2000; D'Angelis et al.,2005, 2007; Teixeira et al., 2016).

Outra possível explicação para a diminuição da concentração de CK no GS seria a presença de vitamina $E$ e de cromo no Tonnus JCR ${ }^{\circledR}$ em pasta. Esses componentes participam no metabolismo energético reduzindo o estresse oxidativo e, consequentemente, o acúmulo das espécies reativas de oxigênio que se depositam na musculatura, podendo reduzir o dano muscular e melhorar a recuperação pós-exercício (Lindinger et al., 2017). Apesar dessa associação ser muito utilizada juntamente com o selênio, estudos apontam resultados poucos satisfatórios (Etchichury, 2004; Kienzle et al., 2006; Vieira et al., 2013).

\section{Conclusão}

A suplementação não interferiu nos parâmetros fisiológicos dos equinos. Houve diminuição da concentração de glicose e de creatina quinase no grupo suplementado, o que indica um melhor aproveitamento da glicose e diminuição da injúria muscular.

\section{Referências}

Becker-Birck M, Schmidt A, Lasarzik J, Aurich J, Möstl E, Aurich $C$. Cortisol release and heart rate variability in sport horses participating in equestrian competitions. J Vet Behav Clin Appl Res. 2013;8(2):87-94.

D’Angelis FHF, Ferraz GC, Boleli IC, Lacerda-Neto JC, Queiroz-Neto A. Aerobic training, but not creatine supplementation, alters the gluteus medius muscle. J Anim Sci. 2005;83(3):579-85.
D’Angelis FHF, Mota MDS, Freitas EVV, Ferraz GC, Abrahão AR, Lacerda-Neto JC, et al. Aerobic Training, But Not Creatine, Modifies Longissimus Dorsi Muscle Composition. J Equine Vet Sci. 2007;27(3):118-22.

Davis BP, Engle TE, Ransom JI, Grandin T. Preliminary evaluation on the effectiveness of varying doses of supplemental tryptophan as a calmative in horses. Appl Anim Behav Sci. 2017;188:34-41.

Ebge-Nwiyi TN, Kalu NA, Naphtali C. Preliminary studies on some haematological and serum biochemical parameters of apparently healthy adult horses in Maiduguri, Nigeria. African J Biomed Res. 2012;15(1):49-53.

Etchichury M. Efeitos da suplementação parenteral com selênio e vitamina $\mathrm{E}$ nos valores hemáticos e séricos de cavalos de enduro [dissertação]. Pirassununga: Universidade de São Paulo; 2004. 85 p.

Feitosa FLF. Semiologia Veterinária - A Arte do Diagnóstico. 3 ed. São Paulo: Roca; 2014. p. 125-32.

Ferraz GC, Teixeira-Neto AR, Pereira MC, Linardi RL, Lacerda-Neto JC, Queiroz-Neto A. Influência do treinamento aeróbio sobre o cortisol e glicose plasmáticos em equinos. Arq Bras Med Vet Zootec. 2010;62(1):23-9.

Franklin S, Allen K. Laboratory exercise testing. In: Hinchcliff KW, Kaneps A, Geor R (EE.). Equine Sports Medicine and Surgery - Basic and clinical sciences of the equine athlete. New York: Saunders; 2014. p. 11-24.

Gillespie Í, Rosenstein PG, Hughes D. Update: Clinical Use of Plasma Lactate. Vet Clin North Am Small Anim Pract. 2017;47(2):325-42.

Gladden LB. Lactate metabolism: a new paradigm for the third millennium. J Physiol. 2004;558(Pt 1):5-30.

Harris PA, Harris RC. Ergogenic potential of nutritional strategies and substances in the horse. Livest Prod Sci 2005;92(2):147-65.

Hassan HY,Aly MA, ElseadyYM, Nayel MA, Elsify AM, Salama AA, et al. The Effect of Race in the Clinical, Hematological and Biochemical Biomarkers in Thoroughbred Horses. Alexandria J Vet Sci 2015;46(1):161-9. 
Hess TM, Rexford J, Hansen DK, Ahrens NS, Harris M, Engle $\mathrm{T}$, et al. Effects of $\Omega-3(\mathrm{n}-3)$ fatty acid supplementation on insulin sensitivity in horses. J Equine Vet Sci. 2013;33(6):446-53.

Hodgson DR, McKeever KH, McGowan CM. The Athletic Horse: Principles and Practice of Equine Sports Medicine. St. Louis, Missouri: Saunders; 2014.

Jordão LR, Rezende ASC, Aquino Neto HM. Suplementação dietética com cromo trivalente em equinos: revisão de literatura. Acta Vet Bras. 2010;4(4):210-4.

Kienzle E, Freismuth A, Reusch A. Double-Blind PlaceboControlled Vitamin E or Selenium Supplementation of Sport Horses with Unspecified Muscle Problems. An Example of the Potential of Placebos. J Nutr. 2006;136(7):2045S-7S.

Lindinger MI, MacNicol JM, Karrow N, Pearson W. Effects of a Novel Dietary Supplement on Indices of Muscle Injury and Articular GAG Release in Horses. J Equine Vet Sci. 2017;48:52-60.

Malblanc A, van Erck-Westergren E. Exercise testing in the field. In: Hinchcliff KW, Kaneps A, Geor R (EE.). Equine Sports Medicine and Surgery - Basic and clinical sciences of the equine athlete. New York: Saunders; 2014. p. 25-42.

Oliveira CAA, Azevedo JF, Miranda ACT, Souza BG, Ramos MT, Costa APD, et al. Hematological and Blood Gas Parameters' Response to Treadmill Exercise Test in Eventing Horses Fed Different Protein Levels. J Equine Vet Sci. 2014;34(11-12):1279-85.

Ostaszewski P, Kowalska A, Szarska E, Szpotański P, Cywinska A, Bałasińska B, et al. Effects of $\beta$-Hydroxy- $\beta$ Methylbutyrate and $\gamma$-Oryzanol on Blood Biochemical Markers in Exercising Thoroughbred Race Horses. J Equine Vet Sci. 2012;32(9):542-51.

Richardson K, Murray JAMD. Fiber for Performance Horses: A Review. J Equine Vet Sci. 2016;46:31-9.

Santiago JM, Almeida FQ Silva LLF, Miranda ACT, Azevedo JF, Oliveira CAA, et al. Hematologia e bioquímica sérica de equinos de concurso completo de equitação em treinamento. Arq Bras Med Vet Zootec. 2013;65(2):383-92.
Sato F, Omura T, Ishimaru M, Endo $\mathrm{Y}$, Murase $\mathrm{H}$, Yamashita E. Effects of daily astaxanthin and L-carnitine supplementation for exercise-induced muscle damage in training thoroughbred horses. J Equine Vet Sci. 2015; 35(10): 836-42.

Schuback K, Essén-Gustavsson B, Persson SG. Effect of creatine supplementation on muscle metabolic response to a maximal treadmill exercise test in Standardbred horses. Equine Vet J. 2000;32(6):533-40.

Stashak TS. Claudicação em Equinos Segundo Adams. 5 ed. São Paulo: Roca; 2006. p. 35-78.

Stefanon B, Bettini C, Guggia P. Administration of branched-chain amino acids to standardbred horses in training. J Equine Vet Sci. 2000;20(2):115-9.

Swirsley N, Spooner HS, Hoffman RM. Supplement Use and Perceptions: A Study of US Horse Owners. J Equine Vet Sci. 2017;59:34-9.

Teixeira FA, Araújo AL, Ramalho LO, Adamkosky MS, Lacerda TF, Coelho CS. Oral creatine supplementation on performance of Quarter Horses used in barrel racing. J Anim Physiol Anim Nutr (Berl). 2016;100(3):513-9.

Trigo P, Castejon F, Riber C, Muñoz A. Use of biochemical parameters to predict metabolic elimination in endurance rides. Equine Vet J Suppl. 2010;(38):142-6.

Urschel KL, Lawrence LM. Amino acids and protein. In: Geor RJ, Harris P, Coenen M (EE.). Equine Applied and Clinical Nutrition. Londres: Saunders; 2013. p. 113-35.

Vetnil. Tonnus JCR. 2012 [acesso 20 nov 2017]. Disponível em: https://tinyurl.com/y86mj8qz.

Vieira WS, Rodrigues IMSMM, Frade NPL, Baldani CD, Botteon RCCM, Botteon PTL. Perfil bioquímico e capacidade antioxidante total em cavalos de polo suplementados com selênio e vitamina-E. Cienc Rural. 2013;43(12):2268-73.

Vincent JB. The Nutritional Biochemistry of Chromium (III). Amsterdam:Elsevier; 2007. p. 110-22.

Votion DM, NavetR, Lacombe VA, Sluse F, Essén-Gustavsson $\mathrm{B}$, Hinchcliff $\mathrm{KW}$, et al. Muscle energetics in exercising horses. Comp Exerc Physiol. 2007;4(3-4):105-18. 